\title{
Where Are the Electromagnetic-wave Counterparts of Stellar-mass Binary Black Hole Mergers?
}

\author{
Shu-Xu Yi ${ }^{1}$ (i) and K. S. Cheng ${ }^{2}$ (i) \\ ${ }^{1}$ Department of Astrophysics, Radboud University Nijmegen, P.O. Box 9010, NL-6500 GL Nijmegen, The Netherlands \\ 2 Pokfulam Road, Department of Physics, the University of Hong Kong, Hong Kong, People's Republic of China \\ Received 2019 August 30; revised 2019 September 12; accepted 2019 September 17; published 2019 October 7
}

\begin{abstract}
Multimessenger astronomy, combining gravitational-wave (GW) and electromagnetic-wave (EM) observations, has a huge impact on physics, astrophysics, and cosmology. However, the majority of sources detected with currently running ground-based GW observatories are binary black hole (BBH) mergers, which disappointingly were expected to have no EM counterparts. In this Letter, we propose that if a BBH merger happens in a gaseous disk around a supermassive black hole, the merger can be accompanied by a transient radio flare like a fast radio burst (FRB). We argue that the total mass and the effective spin derived from GW detection can be used to distinguish such a source from other channels of $\mathrm{BBH}$ mergers. If this prediction is confirmed with future observations, multimessenger astronomy can be greatly improved. The mystery of the origin of FRBs could also be revealed partially.
\end{abstract}

Unified Astronomy Thesaurus concepts: Gravitational waves (678); Radio transient sources (2008); Astrophysical black holes (98)

\section{Introduction}

Since the first gravitational-wave (GW) event detected in 2015, there have been 11 published events (LIGO/Virgo science runs O1-O2; Abbott et al. 2019), and this number is increasing in the current $\mathrm{O} 3$ run. $^{3}$ The observation of the electromagnetic-wave (EM) counterpart of GW170817 offered promising prospects for multimessenger in fundamental physics (e.g., Baker et al. 2017; Nishizawa \& Kobayashi 2018), astrophysics (e.g., Abbott et al. 2018; Wu et al. 2019), and cosmology (Vitale \& Chen 2018). Among the targets of LIGO/ Virgo and other ground-based GW detectors, double neutron star (DNS) mergers, and more rarely neutron star-black hole (NBH) mergers, were the only kinds of sources expected to be accompanied by EM counterparts. This means that the majority of targets, i.e., mergers of stellar-mass binaries black holes (BBHs) were thought to have no hope of being detected via EM. As a result, follow-up searches for EM counterparts are mainly focused on a GW detection that shows a high possibility of being a DNS or a NBH. On the other hand, if some of the $\mathrm{BBH}$ merger can also be detected with EM, multimessenger astronomy can be performed distances one order of magnitude farther than that of DNSs. Sample sizes would also increase significantly.

There are three well-known channels for forming coalescing BBHs: (1) isolated evolution of massive binaries via the common envelope phase (Ivanova et al. 2013; Postnov \& Yungelson 2014); (2) isolated evolution of massive binaries via rotational chemical mixing (de Mink \& Mandel 2016; Mandel \& de Mink 2016; Marchant et al. 2016); (3) dynamical capture in dense clusters (see Chatterjee et al. 2017 and references therein). The abovementioned channels produce BBH mergers in "clean" environments, where no electromagnetic radiation is expected (Liu \& Zhang 2009; Zhang et al. 2016). We refer to those channels as "clean channels" (see Mandel \& Farmer 2018 for a pleasant review). Recently, a new channel of producing

\footnotetext{
https://gracedb.ligo.org/latest/
}

$\mathrm{BBH}$ mergers has received attention, i.e., BBHs embedded in the disk surrounding a supermassive black hole (SMBH), or an active galactic nucleus (AGN). We refer to this channel as "the AGN-disk channel" (Bartos et al. 2017; Stone et al. 2017; Leigh et al. 2018; Yi et al. 2018). In this circumstance, some authors predicted that there would be EM radiation accompanying GW. Farris et al. (2010) found that the shock due to the orbital motion of the binary could provide X-ray radiation. Such radiation may not be luminous enough to be detected from a distance larger than several hundred Mpc. Bartos et al. (2017) suggested there is thermal emission from the transient accretion disk of the $\mathrm{BBH}$ and/or Doppler boosted emission from the relativistic outflow. The fluxes of those high-energy (HE) emissions would be well under the limit of current detectors, unless the accretion rate is highly super-Eddington (we would also like to mention some less-standard scenarios, where gamma-ray radiations are predicted as EM counterparts of BBHs; see Veres et al. 2019). Differing from previous studies that focused on HE radiation, in this Letter we propose that these $\mathrm{BBH}$ mergers will produce flares in the radio bands (several hundred $\mathrm{MHz}$ to $\mathrm{GHz}$ ), like those in fast radio bursts (FRBs) but with a longer duration. The spectral flux densities of such radio flares are above the detecting limit of radio telescopes with large collecting areas. In Section 2, we will present the emission mechanism and make predictions about the EM counterparts.

It is beneficial to ask the following question: how can we distinguish between the AGN-disk channel BBH-originating GW events and ones from clean channels, solely with GW observation? Here we suggest that their total masses and effective spin parameters ( $M_{\text {tot }}$ and $\left.\chi_{\text {eff }}\right)$ can serve as indicators of their origins. The simulation of Postnov \& Kuranov (2019) showed that $\mathrm{BBH}$ mergers from isolated massive binary evolution have $\chi_{\text {eff }}$ clustering around zero. For the dynamical channel, a similar distribution is expected. Meanwhile, the AGN-disk channel BBH has the opportunity to accrete mass and angular momenta from surrounding materials. As a result, we expect them to possess larger $M_{\text {tot }}$ and $\chi_{\text {eff. Indeed, }}$. 


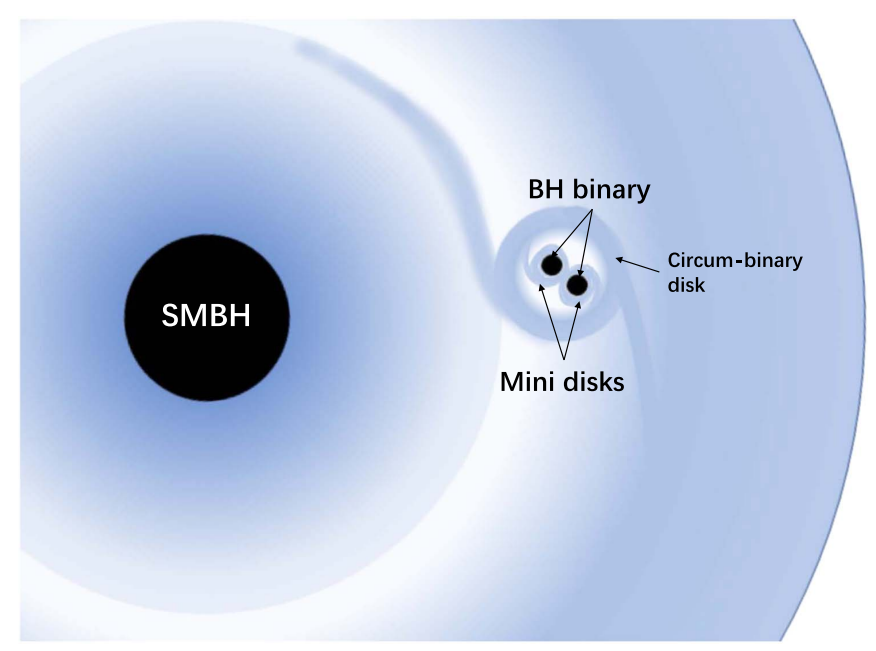

Figure 1. Illustration of a binary black hole system embedded in an accretion disk surrounding a supermassive black hole.

GW170729 has the largest $M_{\mathrm{tot}}$, whose $\chi_{\text {eff }}$ also stands out of the cluster around zero, where others lie. Hence, we suspect that GW170729 could be the first example of AGN-disk channel BBH mergers.

In Section 3, we give the probability density distribution of $M_{\mathrm{tot}}$ and $\chi_{\text {eff }}$ of $\mathrm{BBH}$ mergers that originated from the AGN-disk channel. An analytic formalism and Monte-Carlo simulation are used in this study. In Section 4, we discuss the observational aspects of searching for EM counterparts of GWs from BBHs and their host galaxies. We also discuss caveats of our results. We use geometric units $(G=c=1)$, unless otherwise indicated.

\section{Transient Radio Flares as EM Counterparts of BBH Mergers from the AGN-disk Channel}

The scenario that we are studying in this Letter is the same as that in previous literature: a $\mathrm{BBH}$, either formed in or migrated to and trapped in the disk around a SMBH, inspirals and coalesces with the assistance of accretion from the surrounding matter. As shown in previous numerical simulations, several features are expected (see Figure 1 for an illustration): a region with less dense gas in the AGN disk is cleaned by the BBH. It can be a gap-ring in the orbit where the $\mathrm{BBH}$ lies, or a cavity around the $\mathrm{BBH}$ (Baruteau et al. 2011). Gas inflows through overdense streams from the AGN disk, forming a circumbinary disk (CD). Some of the gas in the $\mathrm{CD}$ is transferred onto individual $\mathrm{BHs}$ via their minidisks. Meanwhile, other gases in the CD are barricaded from being accreted due to the slingshot effect of the binary orbit, which gives rise to an inner gap in the $\mathrm{CD}$.

After the coalescence of the $\mathrm{BBH}$, remnant materials in minidisks and those accumulated in the $\mathrm{CD}$ will be quickly accreted onto the newly formed, highly spinning Kerr BH. The accretion can be transient and temporarily super-Eddington, analogous to that in tidal disruption events (Guillochon \& RamirezRuiz 2013; Kara et al. 2016; Wu et al. 2018) or microquasars (Soria et al. 2014). As proposed in Yi et al. (2019), a transient high-mass transfer onto a stellar-mass $\mathrm{BH}$ from its stellar companion could trigger a clumpy jet, from which FRBs could arise. A similar scenario also applies here: The transient accretion of the remnant gas onto the newly formed Kerr BH can launch an inhomogeneous Blandford-Znajek jet. Gaseous clumpy ejecta in the jet have diverse velocities and are ejected at different instances. Therefore, they might collide among each other at some distance $l_{\mathrm{col}} . l_{\mathrm{col}}$ is related to the time interval between the ejected instances $\delta t$ and the average Lorentz factor of the plasma bulk motion $\gamma$ :

$$
l_{\text {col }} \sim c \gamma^{2} \delta t .
$$

We denote the height of the transient accretion disk at the innermost radius as $h$. The typical separation between clumps being accreted is of the same order of magnitude of $h$. Therefore, $\delta t \sim h / v$, where $v$ is the freefalling velocity at the innermost radius.

The collision between clumps in the jet could trigger plasma oscillation, which will form temporarily charge-separated bunches. The plasma frequency of the oscillation is

$$
\nu_{\mathrm{pls}}(l)=2 \sqrt{\frac{\gamma e^{2} n_{\mathrm{e}}(l)}{\pi m_{\mathrm{e}}}},
$$

where $n_{\mathrm{e}}(l)$ is the number density of electrons, which is simply related to the mass density of the fully ionized plasma $\rho(l)$. Because the dimension of the clump expands along the jet with increasing cone radius, the density of the plasma decreases accordingly as

$$
\rho(l)=\rho_{0}\left(\frac{h}{s(l)}\right)^{3},
$$

where $\rho_{0}$ is the mass density of the gas at the innermost radius, which can be related to the accretion rate with

$$
\dot{M}=2 \pi r_{\text {in }} h \rho_{0} v .
$$

Placing the above equations into Equation (2), we obtain the plasma frequency of the ejecta as a function of the distance to the $\mathrm{BH}$ :

$$
\nu_{\mathrm{pls}}(l)=3.45 \sqrt{\frac{f_{\mathrm{Edd}}}{m \cdot \eta \tilde{h}}} \gamma_{100}^{-2.5}\left(\frac{l_{\mathrm{col}}}{\Theta_{0.1} l}\right)^{1.5} \mathrm{GHz} .
$$

In the above equation, $f_{\text {Edd }}$ and $\eta$ are the Eddington ratio and the radiation efficiency of accretion, respectively.

When such a bunch slides along the curling magnetic field lines in the jet, curvature radiation will be emitted. The frequency of the curvature radiation in the rest frame is

$$
\nu_{\text {cur }}(l)=2 \gamma_{\text {los }} \gamma_{\|}^{3} c / s(l),
$$

where $\gamma_{\text {los }}$ is the Lorentz factor of the bulk motion along the line of sight, and $\gamma_{\|}$is the Lorentz factor corresponding to the sliding of the plasma along the magnetic field lines. For simplicity, we assume $\gamma_{\text {los }} \sim \gamma_{\|} \sim \gamma ; s(l)$ is the local radius of the curvature of the magnetic field lines. Because the magnetic field lines are highly spiral in the jet, we can relate $s(l)$ approximately with the opening angle of the cone of jet as $s(l) \sim \Theta l$.

As a result, the curvature radiation frequency as a function of distance from the $\mathrm{BH}$ is

$$
\nu_{\text {cur }}(l)=3.8 \tilde{h}^{-1} \frac{\gamma_{100}^{2}}{m .} \frac{l_{\text {col }}}{\Theta_{0.1} l} \mathrm{GHz},
$$

where $\tilde{h}$ is the height of the transient accretion disk scaled with the innermost radius $r_{\text {in }}, \gamma_{100}$ is the Lorentz factor of the bulk motion of the bunch scaled with $100, m$. is the mass of the merged $\mathrm{BH}$ in unit of $M_{\odot}$, and $\Theta_{0.1}$ is the opening angle of the jet cone divided by 0.1 . 
When the condition that $\nu_{\text {cur }}=\nu_{\text {pls }}$ occurs at some distance where $l>l_{\text {col }}$, the plasma instability will grow, and the kinetic energy of the bunch will be coherently emitted in a $\mu$ s timescale. It corresponds to a spike in the radio light curve. Each collision among the jet clumps has the opportunity to give such a spike, thus we expect a multicomponent radio flare, which is composed by many spikes, like the sub-structures seen in FRBs. The clumpy jet is thought to arise due to the sudden increase of the accretion rate at the moment of merger. The follow-up accretion is expected to be less temporally varying, and the accretion rate is as high as it is in the onset.

The duration and the power of the flare are proportional to the mass of the $\mathrm{BH}$. The former corresponds to the freefalling timescale near the innermost stable orbit (ISCO):

$$
\tau_{\text {dur }} \sim r_{\mathrm{ISCO}} / c
$$

For a BH with $60 M_{\odot}, \tau_{\text {dur }} \approx 1.8 \mathrm{~ms}$, the apparent luminosity is

$$
L=\frac{8 \eta m \cdot f_{\mathrm{Edd}}}{2 \pi(1-\cos \Theta)} \times 10^{38} \mathrm{erg} \mathrm{s}^{-1} \text {. }
$$

With a typical mass for coalescent $\mathrm{BHs}$ and a super-Eddington accretion rate, $L$ can be $10^{41}-10^{42} \mathrm{erg} \mathrm{s}^{-1}$. Therefore, we expect the EM counterparts to be longer and brighter FRBs than usual. Such FRBs, if localized with high accuracy, are expected to be found at the core regions of active galaxies, and to be simultaneous with a GW chirp. We encourage the reader to refer to Yi et al. (2019) for a more detailed derivation and discussion of the physics of this model.

\section{The Effective Spin and Total Mass of a BBH in the AGN- disk Channel}

The spin evolution of a $\mathrm{BH}$ under accretion along the equatorial plane was first investigated by Bardeen (1970). His result did not take into account the torque exerted by photons emitted from the accretion disk. This term will require a correction to Bardeen's equation, yielding the extreme value of $a^{*}=0.998$ instead of unity (Thorne 1974). We neglect this small correction here. When the angular momentum of the accretion disk is misaligned with the spin of the $\mathrm{BH}$, the evolution of the BH angular momentum is (Perego et al. 2009)

$$
\frac{d \boldsymbol{J}_{\mathrm{BH}}}{d t}=\dot{M} L_{\mathrm{ISCO}} \hat{\boldsymbol{l}}+4 \pi \int_{\mathrm{disk}} \frac{\boldsymbol{L}(R) \times \boldsymbol{J}_{\mathrm{BH}}}{R^{2}} d R,
$$

where $L_{\mathrm{ISCO}}$ is the specific angular momentum brought onto the black hole from ISCO; $\hat{l}$ is a unit vector, which is guaranteed to be parallel with $\boldsymbol{J}_{\mathrm{BH}}$ due to the BardeenPetterson effect (Bardeen \& Petterson 1975); and $\boldsymbol{L}(R)$ is the angular momentum per unit area in the accretion disk at distance $R$ from the $\mathrm{BH}$. The first term on the right is the change rate of the spin modulus, and the second term is the change rate of the spin direction.

Note that

$$
a_{*}= \pm \frac{J_{\mathrm{BH}}}{M^{2}}
$$

where $J_{\mathrm{BH}}$ is the modulus of the spin angular momentum. When the spin is counter-rotating with the binary orbit, the signature of $a_{*}$ is minus. The evolution of $a_{*}$ just follows
Bardeen's equation:

$$
\frac{d a_{*}}{d \ln M}=\frac{1}{M} \frac{L_{\mathrm{ISCO}}}{E_{\mathrm{ISCO}}}-2 a_{*},
$$

where $E_{\mathrm{ISCO}}$ is the specific energy that was brought onto the black hole from ISCO. With the explicit expression of $L_{\mathrm{ISCO}}$ and $E_{\mathrm{ISCO}}$ (Bardeen et al. 1972), the above equation can be integrated to give $a_{*}$ as function of $x$ and initial $a_{*, 0}$, where $x \equiv M_{\mathrm{f}} / M_{0}$ is the ratio between the mass after accretion and the initial mass of the BH. The explicit expression of $a_{*}\left(a_{*, 0}\right.$, $x$ ) is lengthy, thus we just use the numerical integration of Equation (12) for our purposes.

The second term on the right side of Equation (10) governs the alignment of $\mathrm{BH}$ spin and angular momentum of the disk. The angle between the two-vector $\theta$ declines to zero exponentially on an alignment timescale:

$$
\theta(t)=\theta_{0} \exp \left(-\frac{t}{\tau_{\text {align }}}\right)
$$

where $\theta_{0}$ is the initial $\theta$ at $t=0$, and $\tau_{\text {align }}$ is the alignment timescale, which is

$$
\tau_{\text {align }}=1.13 \times 10^{5} \alpha_{0.1}^{58 / 35} f_{\nu_{2}}^{-5 / 7} M_{6}^{-2 / 35}\left(\frac{f_{\text {Edd }}}{\eta_{0.1}}\right)^{-\frac{32}{35}} a_{*}^{5 / 7} \mathrm{yr} .
$$

Because we denote the parallel spin with positive $a_{*}$ and antiparallel spin with negative $a_{*}$, the range of $\theta$ is from $0^{\circ}$ to $90^{\circ}$.

In the above equation, $\alpha_{0.1}$ is the $\alpha$ parameter in a standard thin disk divided by 0.1 (Shakura \& Sunyaev 1973); $f_{\nu_{2}}$ is a nonlinear-effect-related coefficient defined from a simulation; $M_{6}$ is the $\mathrm{BH}$ mass in units of $10^{6} M_{\odot}$ and $\eta_{0.1} \equiv \eta / 0.1$. Those parameters are all of order unity, therefore we set them as one in the following Monte-Carlo simulation.

Equation (9) of $\mathrm{Yi}$ et al. (2018) gives the mass of an individual $\mathrm{BH}$ as a function of its initial mass, orbital separation, and properties of the AGN disk:

$$
m_{\mathrm{f}}=\left(\frac{a_{12,0}^{4} f_{\mathrm{Edd}} \gamma m_{0}^{4 \gamma}}{3.94 \times 10^{-6}}\right)^{1 /(3+4 \gamma)},
$$

where $m_{\mathrm{f}}$ and $m_{0}$ are the masses of the individual $\mathrm{BH}$ in units of $M_{\odot}$, at coalescence and initially respectively; $a_{12,0}$ is the initial orbital separation of the $\mathrm{BBH}$ in units of $10^{12} \mathrm{~cm}$; $f_{\mathrm{Edd}}$ is the Eddington ratio of accretion onto individual BHs during inspiral; $\gamma$ is a parameter used in the numerical simulation of Tang et al. (2017) to describe the mass sink rate. In the above equation, we fix $\eta_{0.1}=1$, thus absorbing any change of $\eta$ into the definition of $f_{\mathrm{Edd}}$. In Yi et al. (2018), the authors studied equal-mass BBHs. Here we inherent this simplification. The total mass is just twice the individual mass at coalescence to be calculated with Equation (15): $M_{\mathrm{tot}}=2 m_{\mathrm{f}} M_{\odot}$.

The effective spin $\chi_{\text {eff }}$ is defined as

$$
\chi_{\mathrm{eff}}=\frac{\left(M_{1} a_{*, 1} \cos \theta_{1}+M_{2} a_{*, 2} \cos \theta_{2}\right)}{M_{\mathrm{tot}}},
$$

where $M_{i}$ and $a_{*, i}(i=1,2)$ are the mass and spin parameter of individual BHs, respectively, and $\theta_{i}$ is the angle between the spin and the orbital angular momenta. 

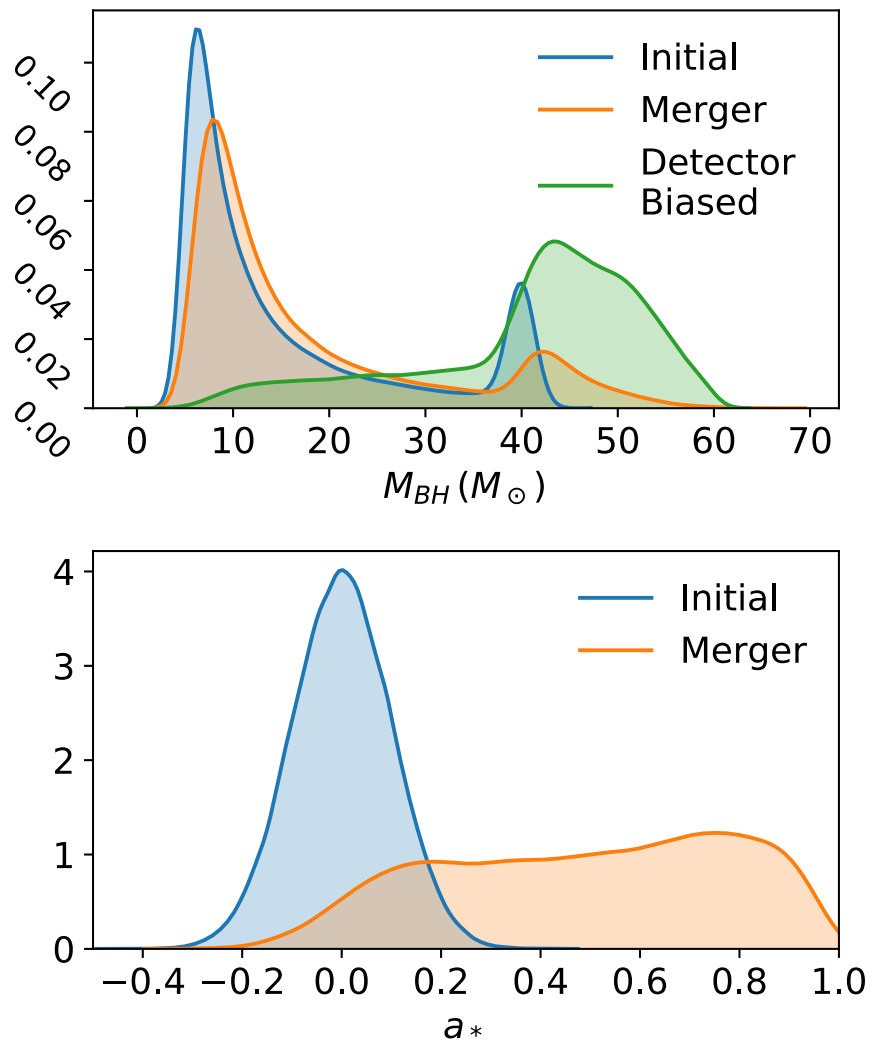

Figure 2. Upper panel: probability distribution masses of individual BHs initially (blue), at merger (orange), and detector-biased (green). Lower panel: probability distribution of spin parameter initially (blue) and at merger (orange).

Given a distribution of initial mass, spin parameters, misalignment angles, Eddington accretion rate of individual BHs, and the initial separation, we can calculate a distribution of the $\chi_{\text {eff }}$ and $M_{\text {tot }}$ at merger using Equations (12)-(16). From a distribution of these parameters, the probability density of $\chi_{\text {eff }}$ and $M_{\text {tot }}$ at merger can be obtained using the Monte-Carlo method.

We assume that the initial mass function of BHs in the AGNdisk channel is the same as that in clean channels. We use a parameterization proposed by Talbot \& Thrane (2018):

$$
p\left(M_{0}\right)=(1-\lambda) p_{\text {pow }}\left(M_{0}\right)+\lambda p_{\text {Gaussian }}\left(M_{0}\right),
$$

where $p_{\text {pow }}$ is a power law with the index $-\alpha$, a low mass cutoff at $M_{\text {gap }}$, and a high-mass cutoff at $M_{\text {cap }} ; p_{\text {Gaussian }}$ is a Gaussian peak. This peak distribution attributes BHs formed via pulsational pair-instability supernovae (PPSNe). We denote the mean and variance of the peak as $m_{\mathrm{pp}}$ and $\sigma_{\mathrm{pp}} . \lambda$ is the portion of PPSNe BHs, which can be estimated through

$$
\lambda=\left(\frac{m_{\text {gap }}}{m_{\text {cap }}}\right)^{\alpha-1} \text {. }
$$

We take the fiducial parameters: $M_{\text {gap }}=5 M_{\odot}, M_{\text {cap }}=40 M_{\odot}$, $\alpha=2, \quad m_{\mathrm{pp}}=M_{\text {cap }}, \quad \sigma_{\mathrm{pp}}=1 M_{\odot}$. The probability density function (PDF) of the initial mass of individual BHs is plotted as a blue line in the upper panel of Figure 2. The distribution of other parameters is as follows: $\gamma$ is calculated from $\tau_{\text {sink }}$ as (Tang et al. 2017)

$$
\gamma=-0.496+1.68 \tau_{\text {sink }}
$$

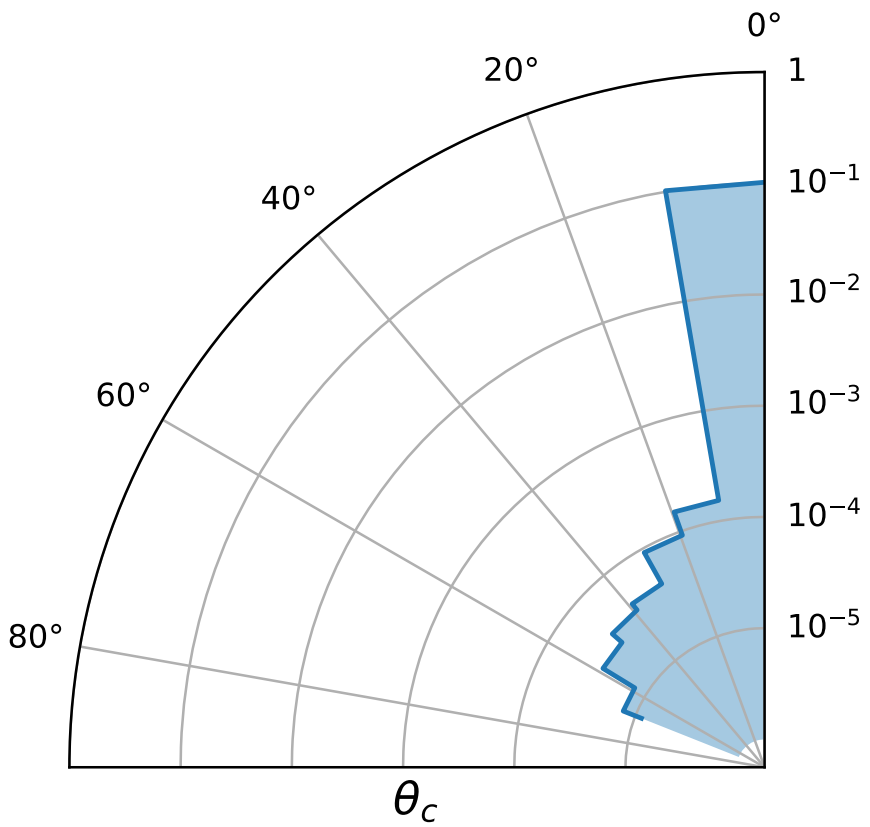

Figure 3. Normalized distribution of misalignment angle $\theta$ of BHs at merger. The radial axis is in logarithm scale.

and $\tau_{\text {sink }}$ is sampled uniformly from 3 to 5 (Yi et al. 2018); $f_{\mathrm{Edd}}$ is sampled uniformly in $\log$ space from -3 to 1 . For the distribution of the binary separation $a_{12,0}$, we use the same value used in Yi et al. (2018), i.e., Öpik's law with an upper limit from ionization of the binary, with the upper limit of $a_{12,0}=5$. The PDF of individual $\mathrm{BH}$ masses at merger is plotted as an orange line in the upper panel of Figure 2. The initial $a_{*}$ of individual BHs is assumed to follow a Gaussian distribution centering at zero and with a width 0.1 , which is plotted together with the pdf of $a_{*}$ at merger in the lower panel of Figure 2. The initial misalignment angle is assumed to be isotropic. Nearly all BHs have their spin axis aligned with the orbital axis at merger, as shown in Figure 3. The time of growth is calculated with

$$
\tau_{\text {growth }}=\log (x) / f_{\text {Edd }} \times 10^{8} \mathrm{yr},
$$

We exclude those samples whose $\tau_{\text {growth }}$ are larger than the typical lifetimes of AGNs, $\tau_{\mathrm{AGN}}$. Here we use a fiducial value of $\tau_{\mathrm{AGN}}=10^{8} \mathrm{yr}$.

We also want to include the effect that the detectable volume has when increasing with the chirp mass of BBH: $V \propto \mathcal{M}^{5 / 2}$ (in the local universe, where $\mathcal{M}$ is the chirp mass of the $\mathrm{BBH}$ and it is proportional to $\left.M_{\mathrm{tot}}\right)$. As a result, the detected distribution will be biased toward higher total mass:

$$
p_{\text {det }}\left(M_{\mathrm{tot}}, \chi_{\mathrm{eff}}\right) \propto p\left(M_{\mathrm{tot}}, \chi_{\mathrm{eff}}\right) M_{\mathrm{tot}}^{5 / 2} .
$$

The detector-biased mass PDF of BHs is plotted as a green line in the upper panel of Figure 2.

In Figure 4, the density plot represents the probability density of detecting an AGN-disk channel BH with $M_{\text {tot }}$ and $\chi_{\text {eff }}$ with GW observation. We also plot the observed data with their uncertainty in the same figure.

It is shown that GW170729 falls far from the cluster of other events in the parameter space, and toward the peak of the predicted distribution of the AGN-disk channel events. This hints that GW170729 is from a different channel. Stone et al. 


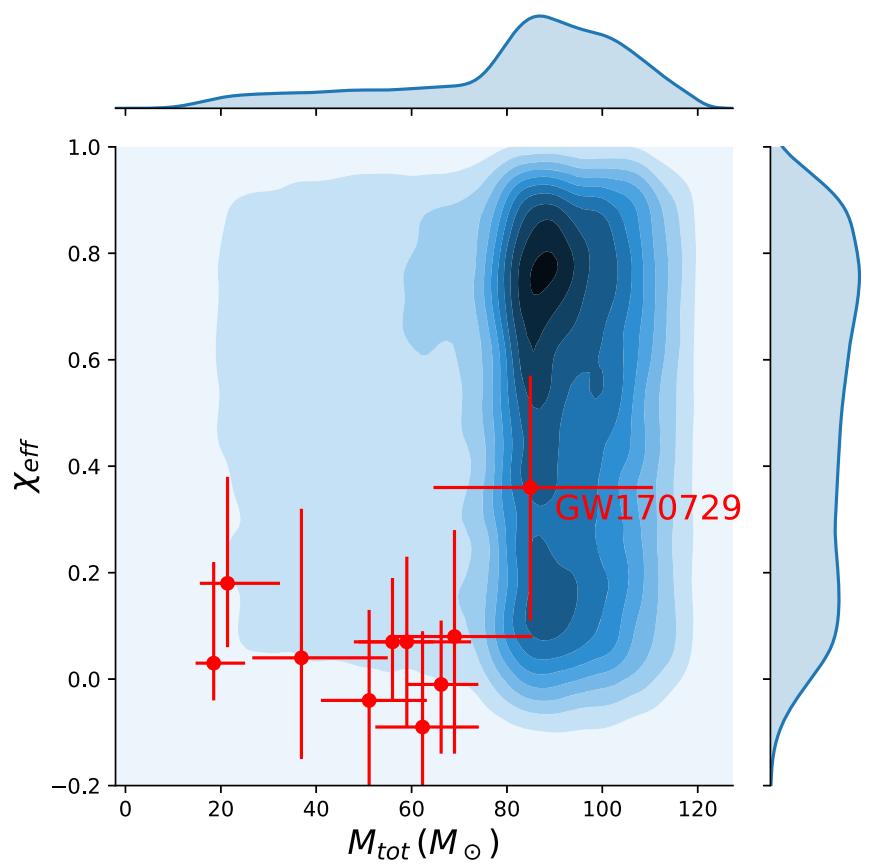

Figure 4. Simulated probability density distribution of the $\mathrm{BBH}$ from AGN disk. The red points with error bars are data from the LIGO/Virgo O1-O2 catalog.

(2017) found that the BBH merger rate density in the AGNdisk channel is $\sim 3 \mathrm{yr}^{-1} \mathrm{Gpc}^{-1}$, with large uncertainty. This is implied to occur among all $\mathrm{BBH}$ merger $\mathrm{GW}$ events; the ratio of AGN-disk events can be a few percent to tens of percent (the $\mathrm{BBH}$ merger rate calculated from the $\mathrm{LIGO} / \mathrm{Virgo} \mathrm{O} 1-\mathrm{O} 2$ runs is 9.7-101 $\mathrm{yr}^{-1} \mathrm{Gpc}^{-1}$ according to Abbott et al. 2019). We suggest a search in the archival data for an FRB around the time of GW170729, and we expect to see some more AGNdisk channel candidates after the $\mathrm{O} 3$ run.

\section{Discussion}

\subsection{Observation of EM Counterparts and Host Galaxies}

It is possible that a FRB will be observed accompanying a GW chirp event, if the BBH merger is viewed face-on. With more GW observatories joining the network, the inclination of the $\mathrm{BBH}$ will be constrained with increasing accuracy. Due to the extreme short duration of the FRB, the GW counterparts are only expected to be detected by chance when the field of view (FoV) of a radio telescope covers the right $\mathrm{GW}$ sources, i.e., $\mathrm{BBH}$ mergers from the AGN channel, with small orbital inclination angles. As the FoVs of FRB monitors are usually large (e.g., Canadian Hydrogen Intensity Mapping Experiment (CHIME) has an FoV of $200 \mathrm{deg}^{2}$ CHIME/FRB Collaboration et al. 2018), it is still likely there to be a simultaneous detection of GW and the FRB.

The candidates of the host galaxy will be chosen from a sample of type II AGNs, from a sky region jointly constrained by the FRB and GW observation, and within a certain redshift range.

\subsection{Caveats}

In Section 3, we demonstrated that compared with $\mathrm{BBH}$ mergers from clean channels, mergers from the AGN channel are more likely to occur toward large $M_{\text {tot }}$ and $\chi_{\text {eff. }}$ Changing the distribution of input parameters will not qualitatively change this conclusion. However, our result cannot be used quantitatively to predict the possibility that a certain GW event is from the AGN-disk channel rather than clean channels. This is because there are large uncertainties in the distribution of parameters determining the $M_{\text {tot }}-\chi_{\text {eff }}$ probability density. The relative ratios among different $\mathrm{BBH}$ formation channels are also not well constrained.

We assumed that the initial mass function and spin distribution of BHs in the AGN channel are the same as those in clean channels. The reality could be different from our assumptions: Yang et al. (2019b) showed that the initial mass function was hardened by the AGN disk due to the orbital alignment process. A non-negligible fraction of $\mathrm{BHs}$ experienced previous mergers (hierarchical mergers), which resulted in higher mass and faster aligned spin (McKernan et al. 2019; Yang et al. 2019a). We hope to include the abovementioned factors in future studies.

S.X.Y. acknowledge support from the Netherlands Organisation for Scientific Research (NWO). K.S.C. is supported by a GRF grant under 17310916.

\section{ORCID iDs}

Shu-Xu Yi (ib https://orcid.org/0000-0001-7599-0174

K. S. Cheng (iD https://orcid.org/0000-0002-8043-9851

\section{References}

Abbott, B. P., Abbott, R., Abbott, T. D., et al. 2018, PhRvL, 121, 161101 Abbott, B. P., Abbott, R., Abbott, T. D., et al. 2019, PhRvX, 9, 031040 Baker, T., Bellini, E., Ferreira, P. G., et al. 2017, PhRvL, 119, 251301 Bardeen, J. M. 1970, Natur, 226, 64

Bardeen, J. M., \& Petterson, J. A. 1975, ApJL, 195, L65 Bardeen, J. M., Press, W. H., \& Teukolsky, S. A. 1972, ApJ, 178, 347 Bartos, I., Kocsis, B., Haiman, Z., \& Márka, S. 2017, ApJ, 835, 165 Baruteau, C., Cuadra, J., \& Lin, D. N. C. 2011, ApJ, 726, 28 Chatterjee, S., Rodriguez, C. L., \& Rasio, F. A. 2017, ApJ, 834, 68 CHIME/FRB Collaboration, Amiri, M., Bandura, K., et al. 2018, ApJ, 863, 48 de Mink, S. E., \& Mandel, I. 2016, MNRAS, 460, 3545

Farris, B. D., Liu, Y. T., \& Shapiro, S. L. 2010, PhRvD, 81, 84008 Guillochon, J., \& Ramirez-Ruiz, E. 2013, ApJ, 767, 25

Ivanova, N., Justham, S., Chen, X., et al. 2013, A\&ARv, 21, 59 Kara, E., Miller, J. M., Reynolds, C., et al. 2016, Natur, 535, 388 Leigh, N. W. C., Geller, A. M., McKernan, B., et al. 2018, MNRAS, 474, 5672 Liu, Y., \& Zhang, S. N. 2009, PhLB, 679, 88

Mandel, I., \& de Mink, S. E. 2016, MNRAS, 458, 2634

Mandel, I., \& Farmer, A. 2018, arXiv:1806.05820

Marchant, P., Langer, N., Podsiadlowski, P., et al. 2016, A\&A, 588, A50

McKernan, B., Ford, K. E. S., O'Shaughnessy, R., et al. 2019, arXiv:1907.04356 Nishizawa, A., \& Kobayashi, T. 2018, PhRvD, 98, 124018

Perego, A., Dotti, M., Colpi, M., et al. 2009, MNRAS, 399, 2249

Postnov, K. A., \& Kuranov, A. G. 2019, MNRAS, 483, 3288

Postnov, K. A., \& Yungelson, L. R. 2014, LRR, 17, 3

Shakura, N. I., \& Sunyaev, R. A. 1973, A\&A, 500, 33

Soria, R., Long, K. S., Blair, W. P., et al. 2014, Sci, 343, 1330

Stone, N. C., Metzger, B. D., \& Haiman, Z. 2017, MNRAS, 464, 946

Talbot, C., \& Thrane, E. 2018, ApJ, 856, 173

Tang, Y., MacFadyen, A., \& Haiman, Z. 2017, MNRAS, 469, 4258

Thorne, K. S. 1974, ApJ, 191, 507

Veres, P., Dal Canton, T., Burns, E., et al. 2019, ApJ, 882, 53

Vitale, S., \& Chen, H.-Y. 2018, PhRvL, 121, 21303

Wu, M.-R., Barnes, J., Martínez-Pinedo, G., et al. 2019, PhRvL, 122, 62701

Wu, S., Coughlin, E. R., \& Nixon, C. 2018, MNRAS, 478, 3016

Yang, Y., Bartos, I., Gayathri, V., et al. 2019a, arXiv:1906.09281

Yang, Y., Bartos, I., Haiman, Z., et al. 2019b, ApJ, 876, 122

Yi, S.-X., Cheng, K. S., \& Luo, R. 2019, MNRAS, 483, 4197

Yi, S.-X., Cheng, K. S., \& Taam, R. E. 2018, ApJL, 859, L25

Zhang, S.-N., Liu, Y., Yi, S., et al. 2016, arXiv:1604.02537 